\title{
The Effect of Nursing Intervention of Postoperative Thirst in Patients after Laparoscopic Cholecystectomy
}

\author{
Wang Xiaolan ${ }^{1,2}$, Liu Cuiqing ${ }^{1,2 . *}$, Zhou Yulan ${ }^{1,2}$, Huang Lu ${ }^{1,2}$ \\ ${ }^{1}$ Department of Hepatobiliary Surgery, The First Affiliated Hospital, Jinan University, Guangzhou, People's Republic of China \\ ${ }^{2}$ School of Nursing, Jinan University, Guangzhou, People's Republic of China
}

Email address:

liueq2018@sina.com (Liu Cuiqing)

${ }^{*}$ Corresponding author

\section{To cite this article:}

Wang Xiaolan, Liu Cuiqing, Zhou Yulan, Huang Lu. The Effect of Nursing Intervention of Postoperative Thirst in Patients after Laparoscopic Cholecystectomy. American Journal of Nursing Science. Vol. 7, No. 3, 2018, pp. 106-108. doi: 10.11648/j.ajns.20180703.14

Received: April 21, 2018; Accepted: May 7, 2018; Published: May 28, 2018

\begin{abstract}
Objective To explore the best method to relieve thirst in patients after laparoscopic cholecystectomy through comparison. Methods 60 patients after undergoing laparoscopic cholecystectomy were randomized into Q-tip group and spraying group. In the Q-tip group, warm boiled water-absorbing Q-tips were applied to embrocate the lips of patients and in the spraying group warm boiled water was sprayed into the oral cavity of the patients once per hour or given when needed within 6 hours after operation so as to compare which method was more effective to relieve thirst. Results Operations were performed well in both groups and there was no postoperative complication. It was shown that warm boiled water spraying achieved better result in relieving thirst 6 hours after operation $(\mathrm{P}<0.05)$. Conclusions Warm boiled water spraying is more effective than warm boiled water-absorbing Q-tip unction to relieve thirst in patients after laparoscopic cholecystectomy.
\end{abstract}

Keywords: Thirst, Post Laparoscopic Cholecystectomy, Warm Boiled Water, Unction, Spraying

\section{Introduction}

Laparoscopic cholecystectomy usually causes discomfort such as thirst due to preoperative fasting and medication. About $62 \%$ of patients after laparoscopic cholecystectomy complain of moderate or severe thirst, and $10 \%$ of them complain of light thirst [1]. Some believe that preoperative and postoperative fasting can be the main cause of postoperative thirst. Although the revised edition of "Practice Guidelines for Preoperative Fasting" by American Society of Anesthesiologists (ASA) in 1999 [2] requires shortening the fasting time, which regulates that patients at any age can be given little transparent liquid containing sugar, the clinical preoperative fasting time is still long for the sake of patients' safety and due to uncertainty of interval time of operation. In comparison with other complications such as bleeding and pain, thirst receives less attention from medical staff. But it has been suggested in some researches that postoperative thirst affects sleep and emotion thus patients' recovery. With the change of medical model, a people-centered care philosophy is emphasized in nursing care which calls for respecting patients' feelings and improving their comfort degree. So much more attention is paid to the postoperative thirst. Medical staff at home and abroad have conducted a series of studies to relieve postoperative thirst by shortening preoperative fasting time, early fasting after operation, preoperative transfusion and improving the temperature and humidity in the ward, etc. Guo Yazhou, et al. promote the function of salivary gland by pressurizing the shuiquan acupoint to relieve postoperative thirst. Clinically, water-absorbing Q-tip is also employed to moisten oral cavity which can to some degree relieve thirst but is time-consuming. And it is risky that the cotton can fall off in the cavity. Therefore, we employed sprinkling can to spray water into the oral cavities of patients after laparoscopic cholecystectomy, which achieved good results in relieving thirst and improving the comfort of oral cavity. The report is as follows:

\section{Data and Methods}

\subsection{General Data}

60 post-laparoscopic cholecystectomy patients admitted to 
our hospital from August 2017 to December 2017 were randomized into experimental group and controlled group, with 30 patients in each group. In the experimental group, the average age was 53.4 with 22 males and 8 females. In the controlled group, the average age was 51.3 with 24 males and 6 females. There was no significant difference in the age between two groups.

\subsection{Inclusion Criteria}

Patients aged from 18 to 65 , without communication disorders, widely awake during anesthesia and complying with the treatment were included. Informed consent was obtained from patients and their family. Patients with dysfunction of heart, lung, liver or kidney, with diabetes or with changed conditions 6 hours after operation were excluded.

\subsection{Methods}

In the controlled group, 3-4 Q-tips with warm boiled water at $38 \sim 40^{\circ} \mathrm{C}$ were given by nurses or families to moisten patients' lips, lingual surface and oral mucosa per hour or when the patients complain of thirst. In the experimental group, 2-3 $\mathrm{ml}$ of warm boiled water in a $10 \mathrm{ml}$-sprinkling can was sprayed onto the sublingual surface, left and right buccal mucosa of the patients who are widely awake from anesthesia. Before spraying, operator should spray the water onto the back of his/her hand twice to discharge the air in the mouth of the sprinkling can. And families should be taught by nurses in advance to use the sprinkling can to spray water.

\subsection{Evaluation Methods}

Visual Analogue Scale (VAS) by National Institutes of
Health is widely recognized because of its convenience, effectivity, ease of management, objectivity and sensitivity [1] It is widely used to evaluate the degree of thirst in adult patients who can express their thirst clearly. So we adopted VAS to evaluate the degree of thirst in the two groups 6 hours after operation. In the scale, 0 means no thirst, 1-4 is light thirst, 5-7 corresponds to moderate thirst, 8-10 indicates severe thirst and 10 means extreme thirst.

\subsection{Statistical Methods}

SPSS20.0 was used to analyze the data, paired-sample $t$ test was adopted to make comparison and chi-square test was for the enumeration data. The data were shown in the form of $\bar{x} \pm s$. When $\mathrm{P}<0.05$, there was significant difference.

\section{Results}

Operations were well performed in both groups and there was no complication. There was significant difference in the thirst relief 6 hours after operation with the result in the experimental group superior to that in the controlled group. The thirst degree in the experimental group was lighter than that in the controlled group and patients above moderate degree in the experimental group were more than those in the controlled group with significant difference. No extreme thirst occurred in both groups as shown in Table 1. In addition, there was no significant difference in the hospital stays and preoperative fasting time $(\mathrm{P}>0.05)$. The average hospitalization stay in experimental and controlled group were $4.61 \pm 0.78 \mathrm{~h}$ and $6.03 \pm 1.43 \mathrm{~h}$ respectively, and preoperative fasting time were $6.03 \pm 1.43 \mathrm{~h}$ and $6.08 \pm 1.2 \mathrm{~h}$ respectively.

Table 1. Thirst relief in both groups.

\begin{tabular}{|c|c|c|c|c|c|c|}
\hline Group & $\operatorname{cases}(n)$ & No thirst & Light thirst & Moderate thirst & Severe thirst & Extreme thirst \\
\hline Controlled group & 30 & 5 & 10 & 10 & 5 & 0 \\
\hline Experimental group & 30 & 15 & 20 & 4 & 1 & 0 \\
\hline
\end{tabular}

chi-square $=7.50, \mathrm{p}=0.006$

\section{Discussion}

Thirst is a kind of feeling craving for water and an important part of the regulatory mechanism of body fluid equilibrium. Perioperative fasting is a regular method to reduce risk of anesthesia in the operation and postoperative complications. Extensive researches at home and abroad have proved that thirst occurs in most patients after operation because of perioperative fasting [3]. Koeppe, A. T. et al. [4] reported 12 patients with 10 having undergone laparoscopic surgery think postoperative dry mouth and thirst are hard to bear in the interview. There are also researches indicating that fasting over 24 hours will lead to dehydration and reduce $90 \%$ of the non-stimulated parotid salivary flow [5]. Preoperative fasting, postoperative fasting within 6 hours and preoperative medication etc. cause thirst in patients after undergoing laparoscopic cholecystectomy. Some people think thirst may affect the sleep quality and increase anxiety in patients, which affects their life quality and thus raises concern from medical staff $[6,7]$. So great efforts were made to find solutions. Epstein, J. B et al. [8] hold that the best method to relieve thirst is to manage and improve the secretion of saliva of patients. Researches abroad have suggested that mouth wash containing glycine betaine, chewing gum and salivary stimulants can stimulate effectively the secretion of saliva to relieve thirst in patients [9-12]. Chen Jian et al. [13] reported that fructus mume spray can promote the secretion of salivary gland to relieve dry mouth and thirst in patients after gastrointestinal operation. There were also reports indicating that finger-press sprayer was more convenient and manageable than traditional moistening tools. Cakir, M [14] used sprinkling can to spray warm boiled water into oral cavity of the patients after general anesthesia to relieve their thirst and achieved obvious effects. Sato, $\mathrm{C}$ et al. proposed that small single-mouth bottle spraying can effectively relieve 
thirst [15]. In the present study, warm boiled water-absorbing Q-tip to moisten lips and sprinkling can to spray warm boiled water into oral cavity can relieve thirst in patients after laparoscopic cholecystectomy. But the latter method achieves better effects and there is significant difference $\left(\chi^{2}=30.276\right.$, $\mathrm{P}<0.05)$. Warm boiled water-absorbing Q-tip can only moisten lips which have no stratum corneum, only a layer of mucous membrane, and thus cannot effectively stimulate the secretion of saliva. Besides, evaporation of water is also a cause of the reduced effect. Some patients need to be moistened every 15-30 minutes, which increases the workload of nurses. What's more, the cotton can easily fall off, and cotton in the oral cavity may lead to danger of aspiration. And 3-4 Q-tips will be cost every time. However, when using sprinkling can, the spray of warm boiled water, characterized by small particles and uniform distribution can not only fully moisten the entire oral mucosa, but also stimulate the secretion of saliva. 2-3 $\mathrm{ml}$ of pray per hour is enough with very few patients requiring increased spray times. It seldom causes cough and aspiration. Its convenience helps the nurses and the families master this method quickly. So it is well received by patients and their families [16].

\section{Conclusions}

Thirst as a symptom has long been thought as the most common clinical complaint in hospitals. We should pay attention to the thirst caused by perioperative fasting and consider it as an important issue to improve the comfort quality of perioperative patients so as to relieve their thirst and promote comfort [17-19]. In the present study, warm boiled water spraying can more effectively relieve thirst in patients after laparoscopic cholecystectomy to improve comfort of oral cavity and thus is worth popularizing.

\section{References}

[1] Guo, Y., Yang, X., Li, Q., \& Li, X. (2010). Pressurization of shuiquan acupoint to relieve thirst after general anesthesia operation. Journal of Nursing Science, 75 (12), 1505-1515.

[2] Osland, E., Yunus, R. M., Khan, S., \& Memon, M. A. (2011). Early versus traditional postoperative feeding in patients undergoing resectional gastrointestinal surgery: a meta-analysis. Jpen Journal of Parenteral \& Enteral Nutrition, 35 (4), 473.

[3] Francisco, S. C., Batista, S. T., \& Gd, P. (2015). Fasting in elective surgical patients: comparison among the time prescribed, performed and recommended on perioperative care protocols, 28 (4), 250.

[4] Koeppe, A. T., Lubini, M., Bonadeo, N. M., Moraes, I., \& Fornari, F. (2013). Comfort, safety and quality of upper gastrointestinal endoscopy after 2 hours fasting: a randomized controlled trial. Bmc Gastroenterology, 13 (1), 158.

[5] Plemons, J. M., Al-Hashimi, I., \& Marek, C. L. (2014). Managing xerostomia and salivary gland hypofunction: executive summary of a report from the american dental association council on scientific affairs. Journal of the American Dental Association, 145 (8), 867-873.
[6] Anil, S., Vellappally, S., Hashem, M., Preethanath, R. S., Patil, S., \& Samaranayake, L. P. (2016). Xerostomia in geriatric patients: a burgeoning global concern. Journal of Investigative \& Clinical Dentistry, 7 (1), 5-12.

[7] Fox, N. F., Xiao, C., Sood, A. J., Lovelace, T. L., Nguyen, S. A., \& Sharma, A., et al. (2015). Hyperbaric oxygen therapy for the treatment of radiation-induced xerostomia: a systematic review. Oral Surgery Oral Medicine Oral Pathology \& Oral Radiology, $120(1), 22-28$.

[8] Epstein, J. B., \& Beier, J. S. (2015). Management of hyposalivation and xerostomia: criteria for treatment strategies. Compendium of Continuing Education in Dentistry, 36 (8), 600-3.

[9] López-Jornet, P., Camacho-Alonso, F., \& Rodriguez-Aguado, C. (2012). Evaluation of the clinical efficacy of a betaine-containing mouthwash and an intraoral device for the treatment of dry mouth. Journal of Oral Pathology \& Medicine, 41(3), 201-206.

[10] Jang, S. Y., Ju, E. Y., Kim, D. E., Kim, J. H., Kim, Y. H., \& Son, M., et al. (2012). First flatus time and xerostomia associated with gum-chewing after liver resection. Journal of Clinical Nursing, 21 (15-16), 2188-2192.

[11] Bryan, G. (2011). Interventions for the management of dry mouth: topical therapies. Cochrane Database of Systematic Reviews, 12 (12), CD 008934.

[12] Pellegrino, F., Groff, E., Bastiani, L., Fattori, B., \& Sotti, G. (2015). Assessment of radiation-induced xerostomia: validation of the italian version of the xerostomia questionnaire in head and neck cancer patients. Supportive Care in Cancer, 23 (4), 925-932.

[13] Chen, J., Lu, Y., \& Wu, F. (2011). Study on experiments and clinical application of dark plum aerosol to relieve xerostomia of postoperative patients after accepting gastrointestinal tract operation. Chinese Nursing Research, 2011, 25 (9):2475-2476.

[14] Cakir, M. (2011). Water and salt metabolism disorders following transsphenoidal pituitary surgery. Turkish Journal of Endocrinology \& Metabolism, 15 (2), 28-32.

[15] Sato, C., Toraiwa, S., Kobayashi, T., Onodera, N., \& Nakamura, S. (2012). Influence of intraoperative fentanyl and remifentanil infusion on early postoperative oral intake after general anesthesia]. Masui the Japanese Journal of Anesthesiology, 61 (9), 1018.

[16] Arai, S. R., Butzlaff, A., Stotts, N. A., \& Puntillo, K. A. (2013). Quench the thirst: lessons from clinical thirst trials. Biological Research for Nursing, 16 (4), 456-66.

[17] Epstein, J. B., \& Beier, J. S. (2015). Management of hyposalivation and xerostomia: criteria for treatment strategies. Compendium of Continuing Education in Dentistry, 36 (8), 600-3.

[18] Plemons, J. M., Al-Hashimi, I., \& Marek, C. L. (2014). Managing xerostomia and salivary gland hypofunction: executive summary of a report from the american dental association council on scientific affairs. Journal of the American Dental Association, 145 (8), 867-873.

[19] Helminen, H., Viitanen, H., \& Sajanti, J. (2009). Effect of preoperative intravenous carbohydrate loading on preoperative discomfort in elective surgery patients. European Journal of Anaesthesiology, 26 (2), 123. 